\title{
APC promoter methylation and protein expression in hepatocellular carcinoma
}

\author{
Antal Csepregi · Christoph Röcken · Juliane Hoffmann · Ping Gu • \\ Stephan Saliger · Oliver Müller · Regine Schneider-Stock · Nadine Kutzner • \\ Albert Roessner - Peter Malfertheiner - Matthias P. A. Ebert
}

Received: 26 November 2006/ Accepted: 24 September 2007/Published online: 1 November 2007

(c) Springer-Verlag 2007

\begin{abstract}
Purpose We investigated the impact of promoter methylation on APC protein expression in patients with hepatocellular carcinoma (HCC).

Materials and methods 50 patients [HCC $(\mathrm{n}=19)$, liver metastasis $(n=19)$, cholangiocellular cancer $(n=7)$, and benign liver tumors $(n=5)$ ] were studied for methylation using Methylight analysis. APC mutation was investigated by protein truncation test and direct sequencing of genomic DNA. The protein expression was evaluated by immunohistochemistry and Western blot analysis.

Results The APC promoter was hypermethylated in $81.8 \%$ of non-cancerous liver tissue samples. All HCC samples and ten patients with liver metastasis (52.6\%) exhibited APC promoter methylation. The degree of
\end{abstract}

A. Csepregi $(\bowtie) \cdot$ J. Hoffmann $\cdot$ P. Gu $\cdot$ S. Saliger .

P. Malfertheiner

Department of Gastroenterology, Hepatology,

and Infectious Diseases, Otto-von-Guericke University,

Leipziger Strasse 44, 39120 Magdeburg, Germany

e-mail: antal.csepregi@medizin.uni-magdeburg.de

C. Röcken

Department of Pathology, Charité University Hospital, Charitéplatz 1, 10117 Berlin, Germany

O. Müller · N. Kutzner

Max-Planck-Institute of Molecular Physiology,

44227 Dortmund, Germany

R. Schneider-Stock $\cdot$ A. Roessner

Institute of Pathology, Otto-von-Guericke University,

Leipziger Strasse 44, 39120 Magdeburg, Germany

M. P. A. Ebert

Department of Medicine II, Klinikum rechts der Isar,

Technical University of München, 81675 Munich, Germany methylation was significantly higher in samples from HCC compared to the non-cancerous liver tissue samples $(63.1 \%$ vs. $24.98 \%$; $p=0.001$ ). The level of APC protein expression was significantly reduced in HCC samples compared to that of the corresponding non-tumor liver tissue $(\mathrm{p}<0.05)$. Conclusions Promoter methylation of the APC gene seems to be of significance in hepatocarcinogenesis and results in reduced protein expression in HCC. Interestingly, APC promoter methylation is also present in the vast majority of non-cancerous liver tissue whose (patho)physiological function remains unresolved.

Keywords Promoter methylation $\cdot A P C$.

Methylight assay $\cdot$ Protein $\cdot$ Hepatocellular carcinoma

\section{Introduction}

Hepatocellular carcinoma (HCC) is the most prevalent primary liver cancer and ranks as the fifth most common cancer in the world, and the third most common cause of cancerrelated death (Bosch et al. 1999). More than 500,000 new cases are diagnosed annually, with an age-adjusted worldwide incidence of 5.5-14.9 per 100,000 population (Llovet et al. 2003). The pathogenesis of HCC is multifactorial. Environmental, infectious, nutritional, metabolic, and endocrine factors contribute directly or indirectly to hepatocarcinogenesis. The importance of individual factors varies geographically depending on environmental and socioeconomic influences. Synchronous occurrence of different risk factors, such as chronic viral hepatitis and aflatoxin burden or iron overload in a single patient or patient population increases the risk of developing HCC. Apart from genomic alterations, epigenetic changes such as promoter methylation, may also contribute to hepatocarcinogenesis. 
Methylation of DNA is a naturally occurring process and involves covalent addition of a methyl group to the $5^{\prime}$ position of the cytosine ring followed by a guanine nucleotide (CpG site) (Bird 1992). Small regions of genomic DNA, called CpG islands, are often located in the promoter region of genes and are usually unmethylated in normal tissue. These regions can be methylated under pathological conditions such as tumor formation. Evidence is increasing that aberrant DNA methylation participates even in the early and precancerous stages of human carcinogenesis.

The adenomatous polyposis coli (APC) tumor suppressor gene encodes a large protein with multiple cellular functions and interactions, including signal transduction in the Wnt-signaling pathway. Preliminary reports suggest that the APC promoter is methylated in up to $81 \%$ of patients with viral hepatitis-induced HCC using the methylation-specific polymerase chain reaction (MSP) (Lee et al. 2003; Yang et al. 2003). Inasmuch as the APC promoter has two major promoters (1A and $1 \mathrm{~B})$ with five alternatively spliced $5^{\prime}$ untranslated regions in different transcripts, so far various groups have reported only methylation of the $1 \mathrm{~A}$ promoter, while the $1 \mathrm{~B}$ promoter has not been shown to be affected by hypermethylation (Tsuchiya et al. 2000; Esteller et al. 2000; Zysman et al. 2002). To date, however, APC protein expression has not been evaluated in these patients. Therefore, we investigated the impact of promoter methylation of the $A P C 1 \mathrm{~A}$ gene promoter on the corresponding protein levels in patients with HCC.

\section{Materials and methods}

\section{Patients}

Tissue samples used in the present study were available from 50 patients who had undergone liver resection. The age of the 19 patients (15 men, 4 women) diagnosed with HCC ranged from 51 to 77 years (mean $67.1 \pm 7.2$ years). Tissue samples from patients with HCC and corresponding non-neoplastic liver parenchyma had been obtained after surgery (mean tumor size $7.95 \pm 5.2 \mathrm{~cm}$ ). The HCCs were categorized according to differentiation into well (G1; three cases), moderately (G2; eleven cases), or poorly (G3; five cases) differentiated types, which correspond, respectively, to Edmondson's Grades I/II, III, or IV (MacSween et al. 2002). In eight males patients, a regular alcohol intake of more than $60 \mathrm{~g} /$ day over a period of 5 years was documented. One patient was diagnosed as having hereditary hemochromatosis. In two cases a positive serology for the core protein of hepatitis B virus (HBV) ("anti-HBc antibody alone") was found without evidence of viral replication. Serological studies for hepatitis $\mathrm{C}$ virus (HCV) remained negative in all patients. Ten patients had cryptogenic HCC (Table 1). Locally advanced disease was found in four cases at time of liver resection. Alpha fetoprotein (AFP) serum level was available from all patients, ranging from 1.3 to $95,468 \mathrm{ng} / \mathrm{ml}$. In eight patients AFP level remained normal during follow-up. Histologically, an advanced liver fibrosis (Ishak's fibrosis scores 3 and 4) or liver cirrhosis (Ishak's fibrosis scores 5 and 6) (Ishak et al. 1995) was diagnosed in 12 patients in the corresponding non-tumor tissue.

Tissue samples were also obtained by surgical resection from 19 patients ( 8 men, 11 women) with liver metastases [colorectal $(n=15)$, gastric $(n=1)$, renal $(n=1)$, and breast $(n=2)$ cancer]. The age of patients ranged from 32 to 82 years (mean $63.5 \pm 14.2$ years). The liver metastases were also categorized according to differentiation into well (G1; no case), moderately (G2; fifteen cases), or poorly (G3; four cases) differentiated carcinomas. Tissue samples were also obtained from seven patients with bile duct $(n=5)$ or gall bladder cancer $(n=2)$ : (G1; one case), moderately (G2; four cases), or poorly (G3; two cases). The age of patients ranged from 42 to 71 years (mean $57.4 \pm 12.2$ years). Five patients (mean age $40.4 \pm 14.6$ years) with benign liver tumors [focal nodular hyperplasia $(n=4)$ or liver adenoma $(n=1)$ ] were also investigated (Table 2). Corresponding non-tumorous liver parenchyma, as confirmed histologically, was also evaluated in all non-HCC cases. This study was approved by the Ethics Committee of the Otto-von-Guericke University of Magdeburg.

\section{DNA extraction}

Genomic DNA was extracted from frozen tissue samples using the proteinase $\mathrm{K}$ digestion as previously reported (Ebert et al. 2003).

Sodium bisulfite modification

Five $\mu \mathrm{g}$ of genomic DNA was heat-denatured for $6 \mathrm{~min}$ at $97^{\circ} \mathrm{C}$, followed by incubation with $0.2 \mathrm{~mol} / \mathrm{l} \mathrm{NaOH}$ for $10 \mathrm{~min}$ at room temperature. The denatured DNA was treated with $3.5 \mathrm{~mol} / \mathrm{l}$ sodium bisulfite and $1 \mathrm{mmol} / \mathrm{l}$ hydroquinone $\left(\mathrm{pH} \mathrm{5.0)}\right.$ for $16 \mathrm{~h}$ at $55^{\circ} \mathrm{C}$. The reaction mixture was purified with a gel extraction kit, and desulfhonated with $0.3 \mathrm{~mol} / \mathrm{l} \mathrm{NaOH}$ for $10 \mathrm{~min}$ at room temperature. The DNA was then precipitated with three volumes of cold ethanol, dissolved in $\mathrm{H}_{2} \mathrm{O}$, and stored at $-20^{\circ} \mathrm{C}$. 


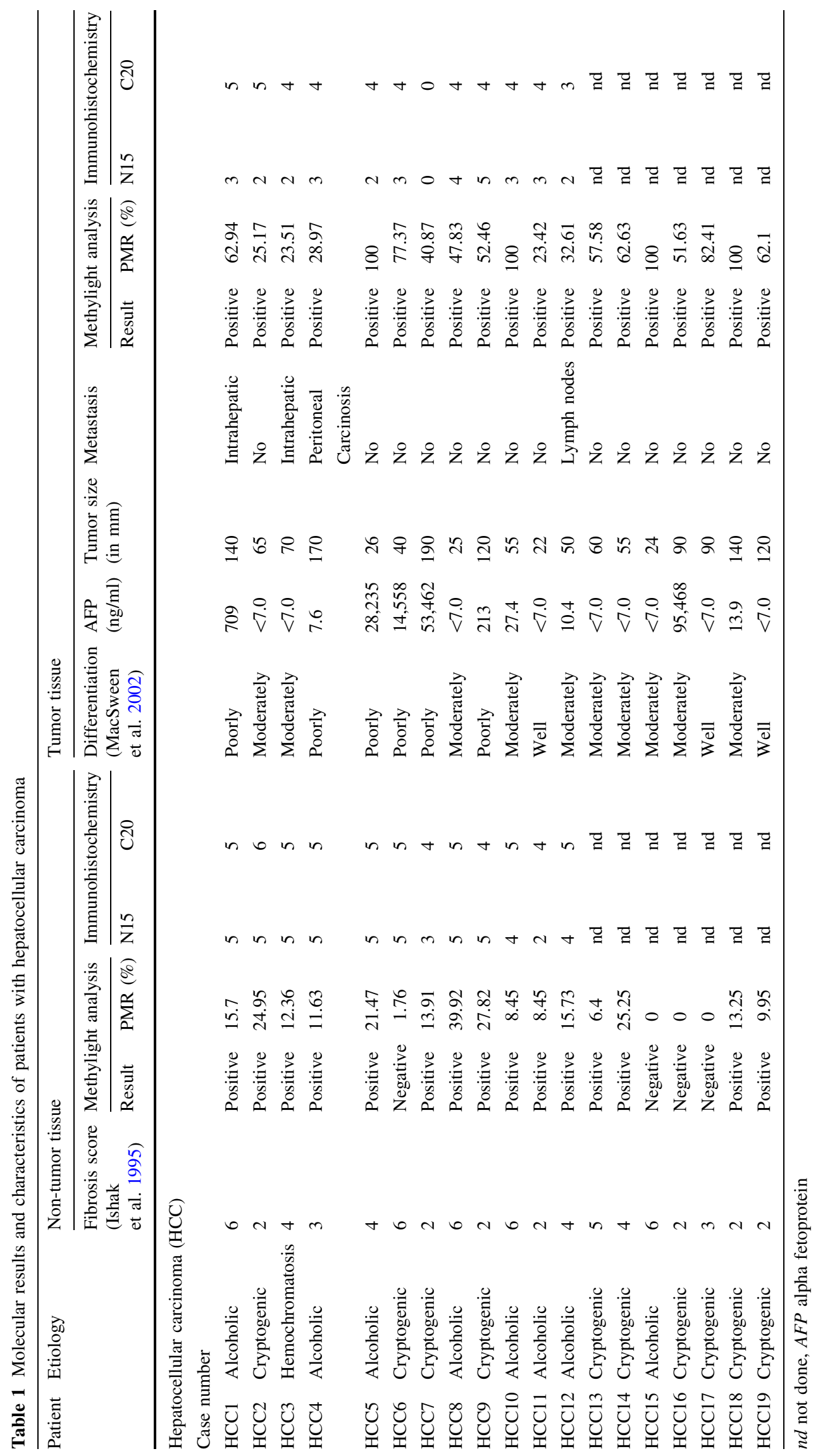


ttattactctccetccacctcCGacatettatactaatcettctaccetaCGaacctcccCGactctttactataCGtatcaactaccatcaacttccttactt actaaaaactaaaacCGCGaaaacataccccCGaaaataCGaaactaaaactaaacaaactataCGattaaaCGaaaccetataccccactaC GaaataCGaatCGaaaaaCGaaaaaaaaacaactatataatcCGctaaataCGaaccaaaaCGctcccattccCGtCGaaaaccCG cCGattaactaaatataaaCGcaCGtaacCGacatataactatattaatacaaccCGccaaaatatcactaaaaacaaaataaaaatactacCGa actCGaaaataaaataaatactaaaaccaccataaccaaacttactaCGaaaaaaaaaaaaaataatttccetCGcactatcttaaacCGataaccttt ccttaacacaaaatccactacaacataccaaaCGaaaaaacaaaaa

Fig. 1 Sequence of the APC 1A Promoter. A portion of bisulfite reverse complement strand of the APC gene promoter (NM_000038) is given with the primer and probe location which were used for the Methylight analysis (underlined and bold). CpG sites are highlighted

Methylight analysis of the APC gene

Genomic DNA of all samples was analyzed by the Methylight technique after bisulfite conversion as previously reported (Eads et al. 2000). In this analysis three oligonucleotids were used in every reactions. Two locusspecific PCR primers flanked an oligonucleotide probe with a $5^{\prime}$ fluorescent reported dye (6FAM) and a $3^{\prime}$ quencher dye (BHQ-1). Oligonucleotide primers and probes were specifically designed to bind to bisulfite-converted DNA. The gene of interest was then amplified and normalized to a reference set $(\beta$-actin $=A C T B)$ to normalize for input DNA. The specificity of the reactions for methylated DNA is confirmed using human unmethylated sperm DNA and CpGenome Universal Methylated DNA [(Chemicon (subsidiary or Serologicals) catalog no. S7821)]. TaqMan PCR reactions were performed in parallel with primers specific for the bisulfite-converted methylated sequence for a particular locus and with the $A C T B$ reference primers. The ratio between the values was calculated in these two TaqMan analyses. The extent of methylation at a specific locus was determined by the following formula: $\left[(\text { gene/actb })^{\text {sample }}:(\text { gene/actb })^{\text {SssI-treatedgenomicDNA }}\right] \times 100$. A cut-off value of $4 \%$ gave the best discrimination between normal and cancerous samples, as previously reported in esophageal cancer (Eads et al. 2000). In our study, samples with a percentage of methylation reference (PMR) of $\geq 4 \%$ fully methylated molecules were termed methylated, whereas samples with a PMR of $<4 \%$ were considered unmethylated. The primer and probe sequences for APC promoter 1A (GeneBank accession number U02509, Fig. 1) were: forward primer $\left(5^{\prime}-3^{\prime}\right)$ : GAACCAAAACGC TCCCCAT; reverse primer $\left(5^{\prime}-3^{\prime}\right)$ : TTATATGT CGGTT ACGTGCGTTTATAT; probe sequence $\left(5^{\prime}-3^{\prime}\right)$ : 6FAMCCCGTCGAAAAC CCGCCGATTA-BHQ1 were used as previously reported (Eads et al. 2000).

\section{Mutation analysis of the APC gene}

The mutation cluster region of the APC was analyzed by a non-radioactive protein truncation test (PTT) in 12 patients with HCC as previously described (Kahmann et al. 2002). Briefly, the region was amplified by PCR from the genomic DNA in two overlapping fragments. The used primers include the sequence motifs, which are necessary for the in vitro transcription and translation, and the sequences, which code for the haemagglutinin (HA) protein motif, which serves as an immuno-tag in the following Western blot detection. The PCR results in two overlapping products: APC I corresponding to sequence position 3283 through 4683 (codon 1089 through 1555) and APC II corresponding to sequence position 4573 through 5970 (codon 1515 through 1984) of the APC gene. Next, the PCR fragments were transcribed and translated in a onetube reaction by using commercial reagents and following the protocol of the supplier (Promega, Mannheim, Germany). The reaction products were separated by electrophoresis on a $12.5 \%$ SDS-polyacrylamide gel and electroblotted. The membrane was incubated with monoclonal anti-HA tag antibody as the first and HRP-coupled goat-anti mouse antibody as the second antibody. Detection was performed by enhanced chemoluminescence (ECL) using commercial reagents. All primer sequences and the exact conditions for PCR and the in vitro transcription and translation are available upon request.

\section{Western blot analysis}

Frozen tissue from non-tumorous liver parenchyma and HCC obtained from three patients were homogenized in lysis buffer containing $1 \mathrm{mM}$ EDTA, $50 \mathrm{mM} \beta$-glycerophosphate, $2 \mathrm{mM}$ sodium orthovanadate, $1 \%$ Triton-100, $10 \%$ glycerol, $1 \mathrm{mM}$ DTT, and protease inhibitors $(10 \mathrm{mg} /$ $\mathrm{ml}$ benzamidine, $2 \mathrm{mg} / \mathrm{ml}$ anti-pain, and $1 \mathrm{mg} / \mathrm{ml}$ leupeptin). The protein concentration of the supernatants was determined by the BCA assay (Bio-Rad, Hercules, CA, USA). Twenty micrograms of protein in each sample was adjusted to Laemmli buffer [2\% SDS, $10 \%$ glycerol, $62.5 \mathrm{mM}$ Tris- $\mathrm{HCl}$ (pH 6.8), $100 \mathrm{mM}$ DTT, and $0.1 \%$ bromphenol blue], denatured by heating at $95^{\circ} \mathrm{C}$ for $5 \mathrm{~min}$, and subsequently separated on $5 \%$ polyacrylamide gels by SDS-gel electrophoresis. After separation, the proteins were transferred onto immunoblot polyvinylidene difluoride 
membrane (Bio-Rad). The membrane was blocked in $5 \%$ skimmed milk in $1 \%$ TBST for $1 \mathrm{~h}$ at room temperature, and then incubated separately with anti-APC-N15 antibody or anti-APC-C20 antibody (Santa Cruz Biotechnology, Santa Cruz, CA, USA) overnight at $4^{\circ} \mathrm{C}$ (dilution 1:200). These two antibodies react with the $\mathrm{N}$-terminal and $\mathrm{C}$-terminal part of the human APC protein, respectively. Membranes were then washed three times in Tris-buffered saline $/ 0.1 \%$ Tween 20 , incubated for $2 \mathrm{~h}$ with peroxidaselabeled anti-rabbit $\operatorname{IgG}(1: 2,500, \mathrm{KPL})$ and diluted in blocking solution. Membrane-bound secondary antibodies were detected by an enhanced chemiluminescence method following the instructions of the manufacturer (ECL plus Western blotting detection reagents, Amersham Biosciences, Piscataway, NJ, USA). The membrane images were evaluated for the quantification of the protein expression, using the GeneGnome and GeneTools image scanning and analysis package (Syngene BioImaging Systems, Synoptics Ltd., Frederick, MD, USA).

\section{Immunohistochemistry}

Tissue samples from HCCs and corresponding nonneoplastic liver parenchyma were studied by immunohistochemistry. Deparaffinized sections were stained using hematoxylin and eosin (H\&E). For immunostaining of paraffin-embedded sections, the slides were deparaffinized in xylol and rehydrated in a graded alcohol series. Endogenous biotin was blocked using the Avidin/Biotin Blocking kit $(2 \times 15 \mathrm{~min}$; Vector Laboratories Inc., Burlingame, CA, USA). Immunostaining was performed with two antibodies directed against human APC (rabbit-antiAPC-N15, dilution 1:200; rabbit-anti-APC-C20, dilution 1:20). Incubation with the primary antibodies was performed in a moist chamber at $37^{\circ} \mathrm{C}$ for $1 \mathrm{~h}$. Gout antirabbit IgG (30 $\mathrm{min}$, room temperature; Vector Laboratories Inc.) served as a secondary antibody. Slides were washed between steps with Tris-buffered saline. Immunoreactions were visualized via an streptavidin-biotin complex, using the Vectastain ABC alkaline phosphatase kit (distributed by CAMON, Wiesbaden, Germany). FastRed (Zytomed, Berlin, Germany) served as chromogen. The specimens were counter-stained with hematoxylin. Omission of primary antibodies served as a negative control.

\section{Immunoreactivity score (IRS)}

For the quantification of the immunohistochemical results, a numerical scoring system was applied. The observed expression of APC in hepatocytes and tumor cells was assessed using two categories. Category A documented the number of immunoreactive epithelial cells as 0 (no immunoreactive cells), $1(<10 \%), 2(11-50 \%)$, and 3 $(>50 \%)$. A positive case was defined as having a category A value of 1 . Category B documented the intensity of the immunostaining as 0 (no immunostaining), 1 (weak), 2 (moderate), and 3 (strong). Finally, the values for category $\mathrm{A}$ and $\mathrm{B}$ were added to construct an "immunoreactivity score" (IRS), which could range from 0 to 6 . Note that the method of calculating the IRS does not allow the individual categories to add up to an IRS of 1 . The immunostaining was performed and analyzed by an experienced GI pathologist (CR) who was blinded to the methylation status of the $A P C$ gene.

\section{Statistical analysis}

The PMR values of the Methylight assays were dichotomized for statistical purposes as reported previously (Eads et al. 2000). The PMR values of tumor and non-tumor samples were compared by Kolmogorov-Smirnov test, and a $P<0.05$ was considered statistically significant.

\section{Results}

$A P C$ promoter methylation in liver cancer

Using the Methylight assay, the methylation status of the IA APC promoter was evaluated in 19 HCCs and the corresponding non-tumor liver samples. All patients with HCC and 15 of the 19 non-tumorous liver samples (79\%) exhibited a PMR $>4 \%$. The mean PMR was 63.10 (range from 23.41 to 100) in the HCC and 14.19 (range from 0 to 39.92 ) in the non-tumor samples (Table 1). The difference between tumor and non-cancerous samples was statistically significant $(P=0.001)$. Even after extraction of the nontumor samples with a PMR $<4 \%$ which were classified as not methylated, the degree of methylation in HCC was significantly higher compared to the non-cancerous tissue samples $(63.1 \%$ vs. $25.12 \% ; P=0.001)$. There was no difference between cirrhotic and non-cirrhotic samples regarding AFP levels, degree of methylation or protein expression evaluated by immunohistochemistry (Table 1). No correlation was found between the size and differentiation of HCC and AFP level, grade of fibrosis or presence of metastasis. Also, the grade of promoter methylation was not significantly different in samples from patients with large tumor or liver cirrhosis compared to those samples obtained from smaller cancers or patients without advanced liver fibrosis (Table 1). 
Table 2 Molecular results and characteristics of patients with other liver tumors

\begin{tabular}{|c|c|c|c|c|c|c|c|}
\hline \multirow[t]{3}{*}{ Patient } & \multirow{3}{*}{$\begin{array}{l}\text { Liver disease/ } \\
\text { primary cancer }\end{array}$} & \multicolumn{4}{|l|}{ Non-tumor tissue } & \multirow{2}{*}{\multicolumn{2}{|c|}{$\frac{\text { Tumor tissue }}{\text { Methylight analysis }}$}} \\
\hline & & \multirow{2}{*}{$\begin{array}{l}\text { Fibrosis score } \\
\text { (Ishak et al. 1995) }\end{array}$} & \multicolumn{2}{|c|}{ Methylight analysis } & \multirow{2}{*}{$\begin{array}{l}\text { Differentiation } \\
\text { (MacSween et al. 2002) }\end{array}$} & & \\
\hline & & & Result & $\operatorname{PMR}(\%)$ & & Result & $\operatorname{PMR}(\%)$ \\
\hline \multicolumn{8}{|c|}{ Cholangiocellular carcinoma (CCC) } \\
\hline \multicolumn{8}{|c|}{ Case number } \\
\hline $\mathrm{CCC} 1$ & Bile duct & 6 & Negative & 2.57 & G2 & Negative & 0 \\
\hline $\mathrm{CCC} 2$ & Bile duct & 0 & Positive & 21.74 & G3 & Negative & 0 \\
\hline $\mathrm{CCC} 3$ & Bile duct & 2 & Positive & 5.82 & G3 & Negative & 0 \\
\hline $\mathrm{CCC} 4$ & Bile duct & 0 & Positive & 16.97 & $\mathrm{G} 2$ & Positive & 5.38 \\
\hline CCC5 & Bile duct & 0 & Positive & 24.38 & $\mathrm{G} 2$ & Negative & 0 \\
\hline CCC6 & Gall baldder & 1 & Negative & 0 & G1 & Negative & 0 \\
\hline $\mathrm{CCC} 7$ & Gall bladder & 0 & Negative & 0 & $\mathrm{G} 2$ & Negative & 0 \\
\hline \multicolumn{8}{|c|}{ Liver metastasis (M) } \\
\hline \multicolumn{8}{|c|}{ Case number } \\
\hline M1 & Breast & 0 & Positive & 9.54 & G2 & Negative & 0 \\
\hline M2 & Colorectal & 0 & Positive & 27.38 & G2 & Negative & 0 \\
\hline M3 & Colorectal & 0 & Positive & 40.77 & G3 & Negative & 0 \\
\hline M4 & Colorectal & 0 & Positive & 26.26 & G3 & Negative & 0 \\
\hline M5 & Nephroblastoma & 0 & Positive & 6.71 & G2 & Negative & 0 \\
\hline M6 & Colorectal & 0 & Positive & 20.91 & G3 & Positive & 6.36 \\
\hline M7 & Colorectal & 4 & Positive & 5.46 & G2 & Negative & 0 \\
\hline M8 & Colorectal & 0 & Positive & 18.23 & G2 & Negative & 2.39 \\
\hline M9 & Colorectal & 0 & Positive & 45.51 & G2 & Positive & 69.95 \\
\hline M10 & Colorectal & 0 & Negative & 0 & G2 & Positive & 23.65 \\
\hline M11 & Gastric & 0 & Positive & 36.68 & G3 & Positive & 23.74 \\
\hline M12 & Colorectal & 0 & Positive & 25.78 & G2 & Positive & 21.65 \\
\hline M13 & Colorectal & 0 & Positive & 12.48 & G2 & Negative & 0 \\
\hline M14 & Colorectal & 1 & Positive & 22.17 & G2 & Positive & 27.73 \\
\hline M15 & Colorectal & 0 & Positive & 29.5 & G2 & Positive & 57.92 \\
\hline M16 & Colorectal & 0 & Positive & 16.95 & G2 & Negative & 0 \\
\hline M17 & Breast & 0 & Positive & 15.85 & G2 & Positive & 30.51 \\
\hline M18 & Colorectal & 0 & Positive & 42.59 & G3 & Positive & 21.44 \\
\hline M19 & Colorectal & 0 & Negative & 0 & G2 & Positive & 7.57 \\
\hline \multicolumn{8}{|c|}{ Benign liver tumor (B) } \\
\hline \multicolumn{8}{|c|}{ Case number } \\
\hline B1 & $\mathrm{FNH}$ & 0 & Negative & 3.52 & na & Positive & 24.09 \\
\hline B2 & FNH & 0 & Positive & 42 & na & Positive & 69.82 \\
\hline B3 & FNH & 0 & Positive & 20.6 & na & Positive & 67.29 \\
\hline B4 & FNH & 0 & Positive & 27.96 & na & Positive & 15.24 \\
\hline B5 & Hepatic adenoma & 0 & Positive & 30.13 & na & Positive & 79.45 \\
\hline
\end{tabular}

FNH focal nodular hyperplasia

$A P C$ promoter methylation in other liver tumors

We then assessed the frequency of $A P C$ promoter methylation in 19 liver metastases and compared it to the corresponding non-tumor liver samples (Table 2). Our analysis showed promoter methylation in eight metastases of colorectal cancers $(50 \%)$, in one gastric and one breast cancer metastasis. Altogether, 52.6\% of metastatic tissue samples were found to be hypermethylated. About 17 of the 19 non-tumor liver samples $(89.5 \%)$ also showed promoter hypermethylation. The mean PMR was 21.19 (range 0-45.51) in the liver metastases and 15.41 (range 0-69.95) in the non-tumor tissue samples. Next, we assessed the frequency of promoter hypermethylation of the $A P C$ gene 
in seven patients with cholangiocarcinoma and found only one tumor with methylated promoter. Four of the seven non-tumor liver samples $(57.2 \%)$ also showed promoter hypermethylation (Table 2). The mean PMR was 10.27 (range 0-24.38) in the non-tumor tissue samples. Nine of the ten tissue samples obtained from patients with benign liver tumors were found to be hypermethylated with a mean PMR of 38.01 (range from 3.52 to 79.45). Altogether, 45 of the 55 non-cancerous liver samples (including also those lesional and extralesional samples obtained from patients with benign liver tumors) exhibited a PMR $>4 \%$ which results in an overall $81.8 \%$ hypermethylation rate. The degree of methylation was significantly higher in samples from HCC compared to the non-cancerous liver tissue samples $(63.10 \%$ vs. $24.98 \% ; P=0.001)$. Using an arbitrarily given PMR of $10 \%, 67 \%$ of non-cancerous liver samples were methylated, and using a PMR of 20 and $30 \%$, only 47 and $20 \%$ of the 55 non-tumor tissues exhibited $A P C$ promoter methylation. Of particular interest was that all HCC samples exhibited a very high PMR above $20 \%$. We found no association of $A P C$ methylation levels and age in any of the cancer patients in either tumor or nontumor tissues.

\section{$A P C$ mutation analysis}

The mutation cluster region of the $A P C$ gene was amplified in two overlapping fragments. The PCR reactions led to products of the expected sizes in 11 of the 12 genomic DNAs (not shown). Sample 9 did not give any PCR product. The PCR products were used as templates in the in vitro transcription and translation reactions and the resulting proteins were analyzed by Western blot. All samples

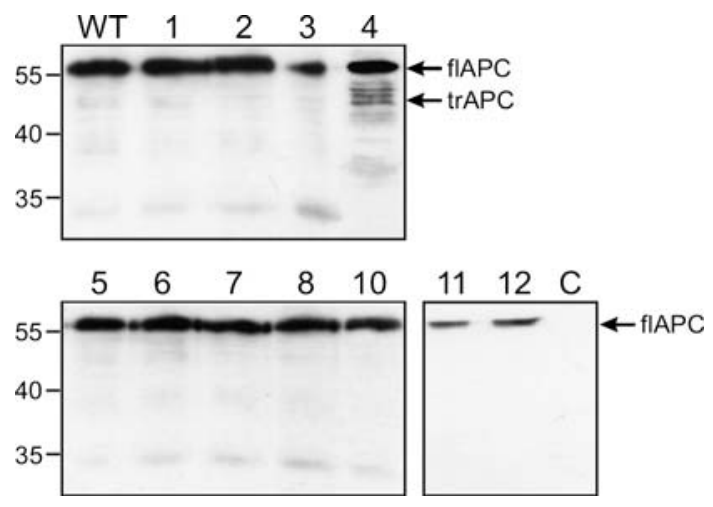

Fig. 2 Western blot of the PTT analysis of the APC protein fragment II (aa1519 through aa1984). The sizes of corresponding marker fragments are shown on the left in kilodaltons. The full-length protein (flAPC II) and the mutated shortened protein (trAPC II) are indicated on the right. WT protein, which was obtained by PTT analysis of nonmutated wild-type DNA, $C$ DNA free control sample

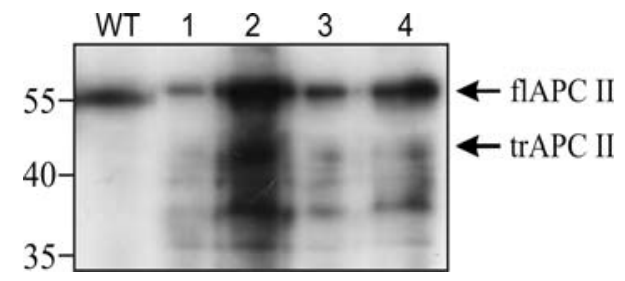

Fig. 3 PTT analysis of four different clones of sample 4 in Fig. 2. The sizes of corresponding marker fragments are shown on the left in kilodaltons. The full-length protein (flAPCII) and the mutated shortened protein (trAPCII) are indicated on the right. All clones show truncated proteins in addition to full length protein. WT protein, which was translated in vitro from non-mutated wild-type DNA

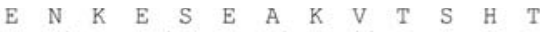

Sbjct: 5675 aaaataaggaatcagaggctaaagttaccagccacacag 5713

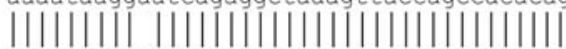

Query: $\quad 94$ aaaataagggatcagaggctaaagttaccagccacacag 132

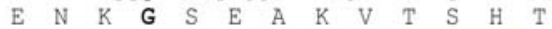

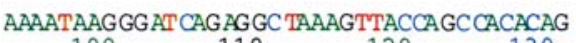

$100 \quad 110 \quad 120 \quad 130$

forward

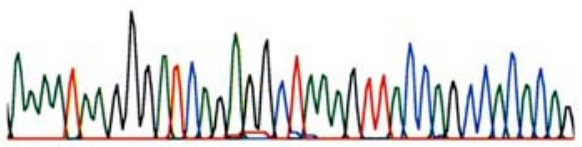

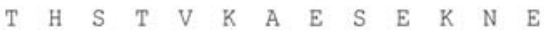

Sbjct: 5713 ctgtgtggctggtaactttagcctctgattccttattt 5675

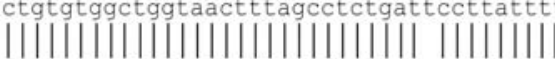

Query: 167 ctgtgtggctggtaactttagcctctgatcccttattt 205

$\begin{array}{cccc}\text { CTGTGTGGCTGGAACTTTAGCCTCTGATCCCTTAITTT } \\ 170 & 180 & 190 & 200\end{array}$

reverse

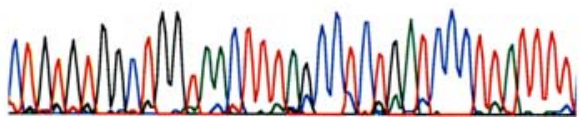

Fig. 4 Sequencing results of the APC gene from sample 4. Upper panel: chromatogram from forward sequencing and blast of the APC gene sequence (GenBank, accession number M74088) using the blast software (http://www.ncbi.nlm.nih.gov/BLAST/). The mutated nucleotide and amino acid are shown in bold. Lower panel: results from reverse sequencing

showed the full-length proteins I and II of the expected size of $\sim 55 \mathrm{kDa}$. Additionally, the analysis of protein II of sample 4 showed fragments, which were $\sim 5-10 \mathrm{kDa}$ smaller than the full-length protein (Fig. 2). The PCR fragment of the sample was cloned. All tested clones showed truncated protein fragments in the PTT. Figure 3 shows the full length protein as a translation product of a wild type control and four different clones. The sequencing of the cloned fragments showed AGGGAT instead of the wildtype sequence AGGAAT (Fig. 4). The A to G transition was found in all tested clones by forward and reverse sequencing. The mutation leads to an alteration of the amino acid Glu to Gly at codon 1889 of the APC protein. 
A

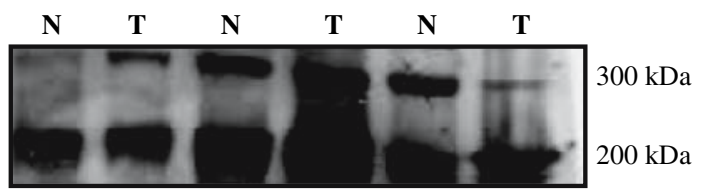

B

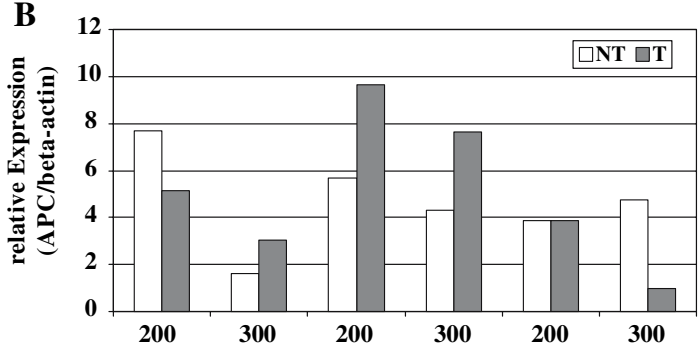

Fig. 5 a Western blot analysis of APC protein expression in three normal $(N)$ and tumor $(T)$ tissue samples obtained from three patients with hepatocellular carcinoma. Two isoforms of the APC protein (300 and $200 \mathrm{kDa}$, respectively) were observed using antibodies directed against the APC protein. The sizes of corresponding marker fragments are shown on the right in kilodaltons. b Quantitative analysis of APC protein expression in hepatocellular cancer. The levels of APC expression in cancer and non-cancer tissues (the order of the patients is identical to the panel above) were standardized to the respective beta-actin expression levels. Overall no significant difference among the two isoforms was observed

Detection of APC protein expression in HCC and liver metastasis

Next we investigated the APC protein expression in both tumor and corresponding non-neoplastic tissue samples obtained from patients with HCC (Fig. 5). By Western blot analysis two isoforms of the APC protein (200 and $300 \mathrm{kDa}$, respectively) were identified. We observed no quantitative differences in APC proteins levels in tumor and non-tumor samples.

Immunohistochemical analysis of APC expression

Immunohistochemistry was performed on paraffin-embedded sections from 14 patients with HCC. APC was found in the cytoplasm of each of the hepatocytes of non-tumorous liver parenchyma (14 patients), bile duct epithelium (Hiltunen et al. 1997), vascular smooth muscle cells (Hiltunen et al. 1997), and tumor cells (Behrens et al. 1998) (Fig. 6). The intensity of immunostaining and the number of immunoreactive cells were decreased in tumorous epithelium of 12 patients, when compared with the corresponding non-tumorous epithelium (Fig. 6). The expression of APC was similar in tumorous and nontumorous epithelium of two patients. The difference between tumor cells, and non-tumorous hepatocytes was further quantified by determining the mean IRS values ( \pm standard deviation). The non-tumorous hepatocytes showed a mean IRS with anti-APC-N15 of $4.5 \pm 1.0$, and with anti-APC-C20 of $4.8 \pm 0.6$, while in HCCs tumor cells showed a mean IRS with anti-APC-N15 of $3.0 \pm 1.5$, and with anti-APC-C20 of $3.8 \pm 1.2$. These IRS values reflect the overall significantly reduced $(P<0.05)$ expression of APC in HCCs.

\section{Discussion}

The human APC gene product is part of a tetrameric complex, composed of conductin, a negative regulator of Wnt signaling, APC, GSK-3 $\beta$ (serine-threonine glycogen synthase kinase $3 \beta$ ) and $\beta$-catenin (Behrens et al. 1998). The intact APC protein normally exerts an anti-tumor effect by forming a tetrameric complex, resulting in the accelerated degradation of $\beta$-catenin and the inhibition of signal transduction to the lympoid enhancer factor- $\mathrm{T}$ cell factor (LEF-TCF) family of transcription factors. The truncated or functionally inactive APC protein which can result from (1) transcriptional silencing through promoter methylation, (2) a point mutation of the $A P C$ gene or (3) loss of heterozygosity (LOH) (Hiltunen et al. 1997; Chen et al. 2005) loses the ability to interact with $\beta$-catenin, allowing the direct interaction of $\beta$-catenin with LEF-TCF family of transcription factors and promoting cells to enter the cell cycle.

The clinical impact of transcriptional silencing of the $A P C$ gene induced by promoter methylation in HCC is currently unclear. A recent study showed that $A P C$ hypermethylation can disrupt the $\mathrm{APC} / \beta$-catenin pathway in primary liver cancer in one-third of the investigated cases (Esteller et al. 2001). In contrast, in an earlier report from China, the promoter region of the $A P C$ gene was found to be free of methylation both in tumor and non-tumor tissue samples obtained from patients with HBV-induced HCC using the same MSP method as reported in the first study (Yu et al. 2002). More recently, three groups used MSP or an MSP-based quantitative methylation analysis and reported that the $A P C$ promoter is methylated in 53-85\% of patients with HCC (Lee et al. 2003; Yang et al. 2003; Lehmann et al. 2005). A particularly high frequency of promoter methylation was found in HCV- and HBV-related HCCs affecting 80 and $55 \%$ of the cases, respectively (Lee et al. 2003; Yang et al. 2003). The $\mathrm{CpG}$ islands of the APC promoter were methylated in up to $27 \%$ of patients with liver cirrhosis and in 14-32\% of liver tissue samples without any pathological changes (Lee et al. 2003; Yang et al. 2003; Lehmann et al. 2005).

In our study, the MethyLight analysis, a novel method for epigenetic studies was used. It provides a more sensitive tool for detecting promoter methylation and also allows quantitative assessment of methylation. We 
Fig. 6 Immunohistochemical expression of APC in liver and hepatocellular carcinomas. The distribution and expression pattern of APC in non-tumorous liver and $\mathrm{HCCs}$ was investigated by immunohistochemistry. Nontumorous epithelium, and tumor were stained with two different anti-APC antibodies (anti-APCN15 and anti-APC-C20). APC was found in the cytoplasm. In the majority of the patients studied, the intensity of immunostaining and the number of immunoreactive cells was decreased in tumorous epithelium (right) when compared with the corresponding non-tumorous epithelium (left)

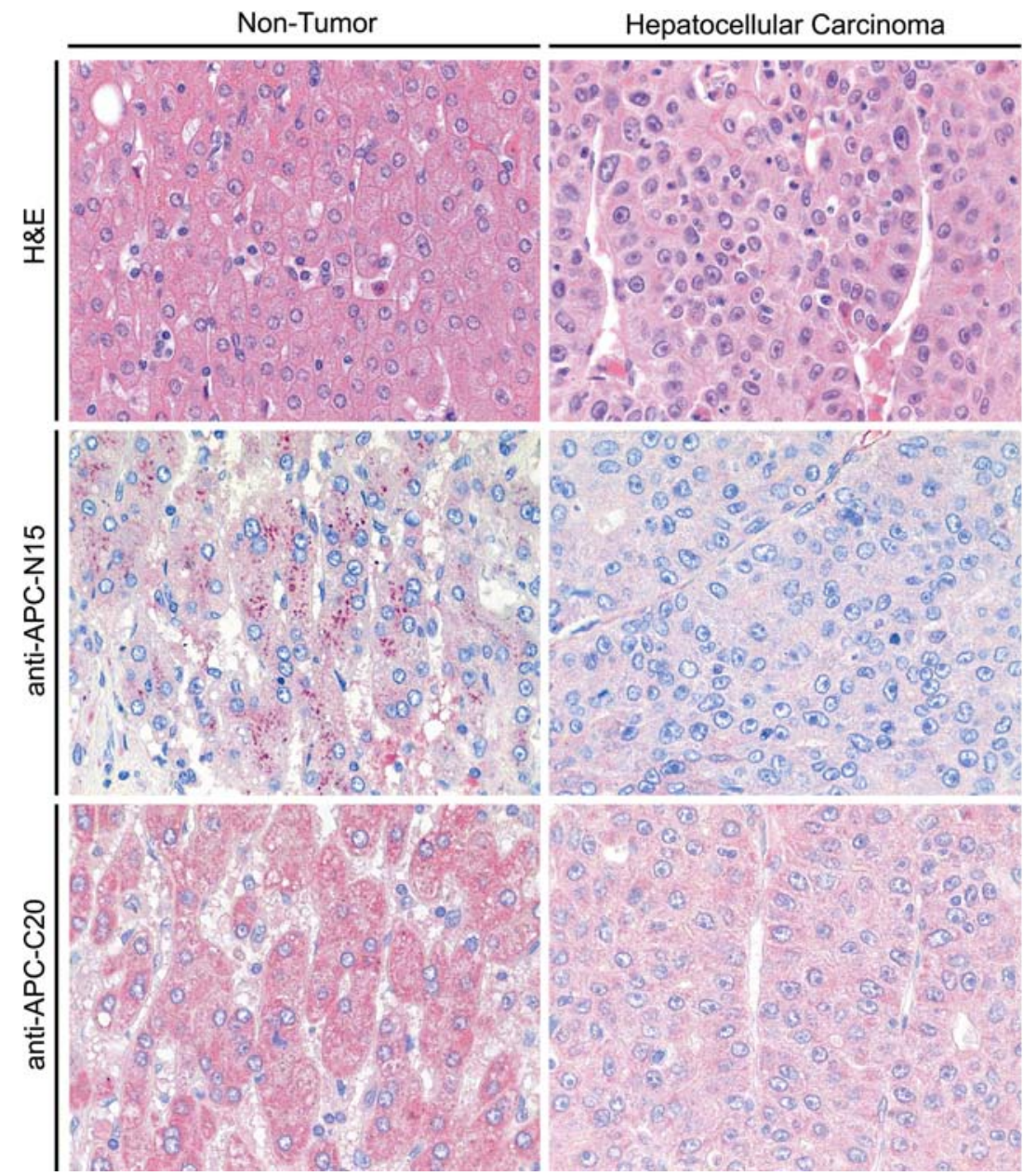

demonstrate a high-methylation rate of the $1 A A P C$ promoter: $81.8 \%$ of the non-cancerous liver tissue samples exhibited a PMR $>4 \%$. Even when a significantly higher methylation rate of $20 \%$ was used, $47 \%$ of samples remained methylated. The (patho) physiological function and significance of the methylated $A P C$ promoter in the non-malignant liver tissue samples is unclear.

The APC promoter has also been reported to be methylated in normal breast tissue samples of women at high risk for breast cancer in $56 \%$ of cases (Lewis et al. 2005). About $97.5 \%$ of patients with non-cancerous gastric mucosa also exhibited $A P C$ promoter methylation and this was considered-in contrast to the colon (Chen et al. 2005) - a normal event (Tsuchiya et al. 2000). However, the function and clinical significance of the methylated $A P C$ promoter in normal tissue and normal mucosa has not been addressed in the past. The high frequency of $A P C$ promoter methylation in non-cancerous liver parenchyma as observed in our study may be explained in different ways. (1) A low level of methylation of the $A P C$ promoter occurs physiologically in the liver, and may have been missed by previous investigation, simply because the
Methylight assay used herein is far more sensitive than the MSP protocol applied by others (Eads et al. 2000); (2) APC promoter methylation in non-tumorous liver parenchyma may result from methylation field effects in the chronically inflamed, fibrotic liver, which bears the risk of developing HCC. Thus, $A P C$ promoter hypermethylation may precede the development of histologically overt lesions, such as dysplastic nodules, small cell dysplasia or HCC, none of which were studied in our non-lesional tissue samples. However, non-tumorous liver parenchyma without evidence of chronic inflammation, fibrosis or cirrhosis (in our study samples obtained from patients with liver metastases) showed APC promoter methylation which supports the first interpretation. Further studies are clearly needed to resolve this very interesting issue in epigenetic studies.

The 1A promoter region of the $A P C$ gene was found to be methylated in all tissue samples from patients with HCC using the $4 \%$ PMR, and all samples remained methylated at a PMR of $20 \%$. Our findings indicate that the $1 \mathrm{~A} A P C$ promoter is generally methylated in the liver suggesting that this epigenetic change is not specific for hepatocarcinogenesis and is not related to chronic hepatitis virus 
infection as reported in the previously published studies (Lee et al. 2003; Yang et al. 2003), because all tissue samples investigated in our study were obtained from patients with non-hepatitis virus-related HCC. It was of particular interest that the methylation grade of the $A P C$ promoter was significantly enhanced in the HCC samples compared with that of the corresponding non-tumor samples. These findings suggest that quantitative epigenetic changes are probably of clinical significance in hepatocarcinogenesis, but further post-transcriptional mechanisms must also be responsible for inactivation of the $A P C$ gene in HCC.

While somatic $A P C$ gene mutations are prevalent in some gastrointestinal tumors such as colorectal cancer (Chen et al. 2005), HCC usually do not harbor APC mutations. In our series, an $A P C$ mutation was found in only one patient $(8 \%)$. The mutation, however, which was found and confirmed in this sample did not cause a stop codon and the sequencing results excluded non-sense or frameshift mutations caused by the Taq polymerase. Nevertheless, the detected transition might be the reason for the truncated proteins which were detected in the PTT. A mutation in a similar repetitive motif of the $A P C$ gene reported by Laken et al. was shown to lead to truncated proteins in the PTT in addition to the full length protein (Laken et al. 1997). The authors explained this finding by failures in the transcriptional or translational machinery caused by the mutation. In summary, $A P C$ gene mutations do not seem to play an important role in the inactivation of $\mathrm{APC}$ in HCC.

Previous studies focused on epigenetic changes of the $A P C$ promoter but did not evaluate the APC protein expression in HCC (Lee et al. 2003; Yang et al. 2003; Esteller et al. 2001; Yu et al. 2002). Therefore, in our analysis, we assessed the impact of promoter methylation of $A P C$ on protein expression in HCC and non-tumor tissue samples. Using Western blot analysis, both isoforms of the APC protein were shown to be present indicating that APC protein is expressed in primary liver cancer cells. In order to further evaluate subcellular expression of the APC protein, immunohistochemistry with a numerical scoring system allowing semiquantitative analysis of the expressed protein was applied. The APC protein was expressed both in non-tumor and tumor tissue samples obtained from patients with HCC, but values derived from our IRS were significantly lower in HCC samples compared with that of non-cancerous liver tissue samples. This finding reflects the overall reduced expression of the APC protein in patients with HCC.

In conclusion, using a highly sensitive tool for detecting epigenetic changes, we demonstrate that $A P C$ promoter methylation is a very frequent epigenetic change in patients with HCC. Furthermore, we show that methylation of the
$A P C$ promoter is also present in the vast majority of the non-tumorous liver tissue samples, indicating that this low level of $A P C$ methylation is universally present in the liver cells and is not a specific marker of liver carcinogenesis and is probably not induced by hepatitis virus infection. Because promoter methylation is significantly enhanced in HCC compared to that of non-tumor samples, and APC expression is also significantly reduced in liver cancer cells, higher degrees of promoter methylation of the $A P C$ gene seem to be responsible for the reduced APC protein expression in HCC. The pathophysiological function of the hypermethylated $A P C$ promoter in non-tumor liver cells deserve further studies to resolve this important issue.

Acknowledgments M. P. Ebert is supported by the a grant from the DFG (187/4-1) and the Heisenberg-Programme (Eb 187/5-1). A. Csepregi is supported by the "Rotationsprogramm" of the NBL-3 of the BMBF.

\section{References}

Behrens J, Jerchow BA, Wurtele M, Grimm J, Asbrand C, Wirtz R, Kuhl M, Wedlich D, Birchmeier W (1998) Functional interaction of an axin homolog, conductin, with beta-catenin, APC, and GSK3beta. Science 280:596-599

Bird A (1992) The essentials of DNA methylation. Cell 70:5-8

Bosch X, Ribes J, Borras J (1999) Epidemiology of primary liver cancer. Semin Liver Dis 19:271-285

Chen J, Rocken C, Lofton-Day C, Schulz HU, Muller O, Kutzner N, Malfertherine P, Ebert MP (2005) Molecular analysis of APC promoter methylation and protein expression in colorectal cancer metastasis. Carcinogenesis 26:37-43

Eads CA, Lord RV, Kurumboor SK, Wickramasinghe K, Skinner ML, Long TI, Peters JH, DeMeester TR, Danenberg KD, Danenberg PV, Laird PW, Skinner KA (2000) Fields of aberrant CpG island hypermethylation in Barrett's esophagus and associated adenocarcinoma. Cancer Res 60:5021-5026

Ebert MP, Yu J, Hoffmann J, Rocco A, Rocken C, Kahmann S, Muller O, Korc M, Sung JJ, Malfertheiner P (2003) Loss of betacatenin expression in metastatic gastric cancer. J Clin Oncol 21:1708-1714

Esteller M, Corn PG, Baylin SB, Herman JG (2001) A gene hypermethylation profile of human cancer. Cancer Res 6:32253229

Esteller M, Sparks A, Toyota M, Sanchez-Cespedes M, Capella G, Peinando MA, Gonzalez S, Tarafa G, Sidransky D, Meltzer SJ, Baylin SB, Herman JG (2000) Analysis of adenomatous polyposis coli promoter hypermethylation in human cancer. Cancer Res 60:4366-4371

Hiltunen MO, Alhonen L, Koistinaho J, Myohanen S, Paakkonen M, Marin S, Kosma VM, Janne J (1997) Hypermethylation of the APC (adenomatous polyposis coli) gene promoter region in human colorectal carcinoma. Int J Cancer 70:644-648

Ishak K, Baptista A, Bianchi L, Callea F, De Groote J, Gudat F, Denk H, Desmet V, Korb G, MacSween RN (1995) Histological grading and staging of chronic hepatitis. J Hepatol 22:696699

Kahmann S, Herter P, Kuhnen C, Muller KM, Muhr G, Martin D, Soddemann M, Muller O (2002) A non-radioactive protein truncation test for the sensitive detection of all stop and frameshift mutations. Hum Mutat 19:165-172 
Laken SJ, Petersen GM, Gruber SB, Oddoux C, Ostrer H, Giardiello FM, Brensinger JD, Vogelstein B, Kinzler KW (1997) Familial colorectal cancer in Ashkenazim due to a hypermutable tract in APC. Nat Genet 17:79-83

Lee S, Lee HJ, Kim JH, Lee HS, Jang JJ, Kang GH (2003) Aberrant $\mathrm{CpG}$ island hypermethylation along multistep hepatocarcinogenesis. Am J Pathol 163:1371-1378

Lehmann U, Berg-Ribbe I, Wingen LU, Brakensiek K, Becker T, Klempnauer J, Schlegerberger B, Kreipe H, Flemming P (2005) Distinc methylation pattern of benign and malignant liver tumors revealed by quantitative methylation profiling. Clin Cancer Res 11:3654-3660

Lewis CM, Cler LR, Bu DW, Jochbauer-Muller S, Milchgrub S, Naftalis EZ, Leitch AM, Minna JD, Euhus DM (2005) Promoter hypermethylation in benign breast epithelium in relation to predicted breast cancer risk. Clin Cancer Res 11:166-172

Llovet JM, Burroughs A, Bruix J (2003) Hepatocellular carcinoma. Lancet 362:1907-1917
MacSween RNM, Burt AD, Portman BC (eds) (2002) Pathology of the liver. London Churchill Livingstone, Edinburgh

Tsuchiya T, Tamura G, Sato K, Endoh Y, Sakata K, Jin Z et al (2000) Distinct methylation patterns of two APC gene promoters in normal and cancerous gastric epithelia. Oncogene 19:3642-3646

Yang B, Guo M, Herman JG, Clark DP (2003) Aberrant promoter methylation profiles of tumor suppressor genes in hepatocellular carcinoma. Am J Pathol 163:1101-1107

Yu J, Ni M, Xu J, Zhang H, Gao B, Gu J, Chen J, Zhang L, Wu M, Zhen S, Zhu J (2002) Methylation profiling of twenty promoter$\mathrm{CpG}$ islands of genes which may contribute to hepatocellular carcinogenesis. BMC Cancer 2:29

Zysman M, Saka A, Millar A, Knight J, Chapman W, Bapat B (2002) Methylation of adenomatous polyposis coli in endometrial cancer occurs more frequently in tumors with microsatellite instability phenotype. Cancer Res 62:3663-3666 